\section{Efecto del gel del extracto liofilizado de Croton lechleri sobre el tejido pulpar en pulpotomías realizadas en Oryctolagus cuniculus}

\section{Croton lechleri lyophilized extract gel effect on Oryctolagus cuniculus ' pulp tissue}

\begin{abstract}
Resumen
Objetivo. Determinar el efecto del gel del extracto liofilizado de Croton lechleri sobre el tejido pulpar radicular en pulpotomías realizadas en Oryctolagus cuniculus. Métodos. Se utilizaron 32 conejos machos de raza neozelandesa de dos meses de edad que se asignaron aleatoriamente en cuatro grupos de ocho animales: control, formocresol, Croton lechleri $1 \%$ y Croton lechleri $2,5 \%$. En cada animal se trabajaron cuatro pulpotomías. Se analizaron variables clínicas (presencia de abscesos, movilidad dentaria e infecciones) e histopatológicas (formación de fibras, cantidad de células proinflamatorias y cantidad de células regenerativas) al cabo de un día, 7, 15, y 30 días. Resultados. En la evaluación clínica, en todos los tiempos evaluados, no se evidenció la existencia de abscesos submucosos, fistula o movilidad dentaria en ninguno de los grupos de tratamiento. En la evaluación histopatológica a 1 día, la presencia de capilares fue abundante en todas las muestras del grupo control y en la mitad de las muestras del grupo formocresol, mientras fue moderada en todas las muestras de los grupos Croton lechleri 1\% y 2,5\%. A los 7 , 15 y 30 días, la presencia de capilares fue mayor en el grupo formocresol. En todos los tiempos evaluados en el grupo Croton lechleri 2,5\%, evidenció menor número de células inflamatorias (macrófagos y linfocitos), mayor cantidad de células de reparación (fibroblastos, odontoblastos) y fibras colágenas normales. Conclusiones. El gel del extracto liofilizado de Croton lechleri 2,5\% evidenció mayor efecto antiinflamatorio, cicatrizante y regenerador sobre el tejido pulpar en comparación a los otros tratamientos.
\end{abstract}

Palabras clave: Croton; Pulpotomía; Cicatrización; Regeneración (fuente: DeCS BIREME).

\begin{abstract}
Objective. To determine the effect of the Croton lechleri lyophilized extract gel on root pulp tissue of pulpotomies performed on Oryctolagus cuniculus. Methods. Thirty-two two-month-old male New Zealand rabbits race, were randomly assigned into four experimental groups: a) control, b) formocresol, c) 1\% Croton lechleri and d) $2.5 \%$ Croton lechleri. Four pulpotomies were done on each animal. Clinical variables (abscesses, tooth mobility and infections) and histopathological variables (fiber formation, pro-inflammatory cell and regenerative cell number) were analyzed after $1,7,15$, and 30 days. Results. No clinical signs were evident in all experimental groups evaluation at any time period. In the first day histopathological evaluation, there were abundant presence of capillaries in all control group samples and half of formocresol ones, while $1 \%$ Croton lechleri and $2.5 \%$ Croton lechleri groups, had a moderate number in all samples. In the 7,15 and 30 evaluation days, the number of capillaries was
\end{abstract}

\section{Artículo Original}

Eufemia Isabel Correa-Olaya ${ }^{1, a}$, Víctor Manuel Chumpitaz Cerrate $^{1, b}$, María Teresa del Pilar Chu Morales ${ }^{2, c}$

${ }^{1}$ Universidad Nacional Mayor de San Marcos, Facultad de Odontología, Lima, Perú.

2 Universidad de San Martín de Porres, Facultad de

Odontología, Lima, Perú.

a Maestra en Docencia e Investigación en Estomatología.

${ }^{b}$ Doctor en Ciencias de la Salud.

c Doctor en Salud Pública.

\section{Correspondencia:}

Eufemia Correa: ecorreao@unmsm.edu.pe

Av. San Borja Norte N¹101-101. San Borja, Lima- Perú. Código postal: 051.

ORCID: 0000-0001-6510-1038

\section{Coautores:}

Víctor Manuel Chumpitaz Cerrate: vchumpitaz@unmsm. edu.pe

ORCID: 0000-0001-7073-8076

María Teresa del Pilar Chu Morales: pilichum@yahoo.es ORCID: 0000-0001-9409-7656

\section{Editora:}

Antonieta Pérez-Flores

Universidad de Concepción, Chile

Conflicto de intereses: los autores declaran no tener conflictos de interés.

Fuente de financiamiento: autofinanciado.

Recibido: 10/11/20

Aceptado: 18/01/21

Publicado: 01/07/21 
higher in formocresol group than the others. The $2.5 \%$ Croton lechleri group, showed a lower number of inflammatory cells (macrophages and lymphocytes), a higher number of repair cells (fibroblasts, odontoblasts) and normal collagen fibers than the others, over all evaluated times. Conclusions. The $2.5 \%$ Croton lechleri lyophilized extract gel showed a greater anti-inflammatory, healing and regenerating effect on the pulp tissue compared to the other experimental groups.

Keywords: Croton; Pulpotomy; Wound healing; Regeneration (source: MeSH NLM).

\section{Introducción}

En el Perú, las enfermedades de la pulpa dental se encuentran entre las diez primeras causas de morbilidad en la consulta dentalentre la población infantil, ocupando el sétimo lugar, y considerándose un problema de salud pública ${ }^{1}$. A medida que la infección avanza, también aumenta la destrucción del tejido pulpar, llegando a un estado de pulpitis reversible, en estas situaciones y considerando que el diente aún se encuentra lejos de su etapa de exfoliación, es recomendable la pulpotomía ${ }^{2}$.

Los biomateriales utilizados en los procedimientos de pulpotomía poseen diferentes propiedades que participan en distintos grados en laregeneración del complejo dentino pulpar, siendo en algunos de estos materiales unadesventaja,su alto costo de adquisición. Dentro de estos biomateriales, el agregado trióxido mineral (MTA) evidencia buenos resultados clínicos e histológicos ${ }^{3}$, considerándose como una alternativa al formocresol tradicional, al formar un puente dentinario delgado. Sin embargo, su costo es elevado, presentando un tiempo de fraguado extenso, de aproximadamente4 horas ${ }^{4}$. Otro biomaterial en uso es el sulfato férrico, que presenta resultados similares al formocresol. Por otra parte el silicato tricálcico, por sus estudios clínicos y radiográficos favorables, podría ser el material de primera elección en el futuro, si el costo del producto fuera menor y la mezcla fuese manual ${ }^{5}$.

La literatura concuerda que el biomaterial «ideal» para el tratamiento de pulpotomía todavía no ha sido encontrado, ya sea por los elevados costos que presentan algunos, por las desventajas de otros o por los resultados cuestionables de algunos ${ }^{3,4}$. Por ello, en la presente investigación se decidió investigar un nuevo biomaterial a base de la "sangre de grado", ya que el Croton lechleri Mull. $A r g$ (nombre científico de la planta) es una especie de la Amazonía peruana, muy empleada e investigada. $\mathrm{Ha}-$ llazgos científicos confieren que presenta propiedades cicatrizantes, antivirales, antiinflamatorias, antioxidantes, antibacterianas y antiparasitarias, entre otras. Contiene como principal componente la "taspina", un alcaloide con propiedades antiinflamatorias y cicatrizantes ${ }^{6,7}$ que induce el desarrollo de fibroblastos y colágeno ${ }^{8}$. Esta planta ha sido utilizada de forma empírica por los pueblos oriundos como un regenerador de heridas.

El presente estudio pretende contribuir, uniendo la ciencia antigua y la medicina de tradición, a los conocimientos de la Odontopediatría, permitiendo un avance en la creación de un nuevo material dental bajo una fórmula farmacéutica de uso tópico, con escasos efectos adversos, inocuo para los tejidos al aplicarse directamente sobre el tejido pulpar, presentando un menor tiempo de aplicación, fácil manipulación, bajo costo comparado con los otros materiales disponibles en el mercado, y con resultados clínicos e histológicos favorables.

El objetivo de este estudio fue determinar el efecto clínico e histopatológico del gel del extracto liofilizado de Croton lechleri sobre el tejido pulpar radicular en pulpotomías realizadas en Oryctolagus cuniculus.

\section{Métodos}

Se realizó un diseño experimental en 32 Oryctolagus cuniculus machos vivos, de raza neo zelandeza de 2 meses de edad y peso entre 1,1 a 1,5 Kg. Se controló la variable independiente de la apertura y exéresis de la pulpa cameral, se dividió la muestra en aplicaciones de formocresol, gel del extracto liofilizado de Croton lechleri $1 \%$ y $2,5 \%$ para estudiar su eficacia en pulpotomías. Los animales fueron obtenidos del Instituto Nacional de Salud (Lima, Perú). Se excluyeron aquellos con alguna enfermedad o alteración que modifiquen el desarrollo del estudio, o que la hubiesen adquirido durante la realización del mismo.

Adecuación de los animales. Los animales fueron pesados en una balanza calibrada e inmovilizados en un cepo para conejos, se procedió a depilar la superficie dorsal de la oreja derecha con crema depilatoria. A continuación, se aplicó dos gotas de xilol sobre la porción más distal de la vena marginal de la oreja del conejo para dilatarla y facilitar la administración intravenosa. Se realizó la asepsia y antisepsia de la zona de punción e inmediatamente se administró Ketamina $50 \mathrm{mg} / \mathrm{mL}$ a dosis de $20 \mathrm{mg} / \mathrm{kg}$ por vía intravenosa lenta. Luego de $20 \mathrm{~s}$ de la administración de Ketamina se verificó la pérdida de la conciencia, a los $40 \mathrm{~s}$ la disociación y a los $60 \mathrm{~s}$ la analgesia. Los conejos fueron marcados en la región dorsal de la oreja izquierda con un plumón indeleble, indicando el grupo al cual eran asignados. A: grupo apertura y exéresis de la pulpa cameral (control), B: grupo formocresol, C: grupo Croton lechleri 1\%, D: grupo Croton lechleri 2,5\%.

Obtención del extracto y gel. Para la elaboración del gel de Croton lechleri se recolectaron $500 \mathrm{~mL}$ de muestra "látex de sangre de grado" procedente del Departamento de Tarapoto y se almacenó en un recipiente ámbar protegido de la luz. Se confirmó la identidad de Croton lechleri, en el Museo de Historia Natural de la Universidad Nacional Mayor de San Marcos. Confirmada la 
identidad de Croton lechleri, se liofilizaron $500 \mathrm{~mL}$, y se obtuvo $29 \mathrm{~g}$ de látex liofilizado sin aditivo de cristales amorfos de color rojizo. Finalmente se procesó la humedad por método USP41 obteniendo un \% humedad = $10,11 \%$, y en la cuantificación de alcaloides por volumetría en medio no acuoso se encontró un porcentaje deTaspina $=4,7 \%$. Estos procedimientos fueron realizados en la Facultad de Farmacia y Bioquímica, Laboratorio Cenprofarma - Centro de Control Analítico.

La confirmación del gel se realizó a través de prueba de solubilidad, colocando en 5 tubos de ensayo aproximadamente $0,5 \mathrm{mg}$ de Croton lechleri liofilizado y se adicionó $5 \mathrm{~mL}$ de solvente: cloroformo, propilenglicol, alcohol etílico $96^{\circ}$, alcohol absoluto, y alcohol absoluto con propilenglicol (el más soluble). Posteriormente en un vaso Becker de $100 \mathrm{~mL}$ se colocó $1 \mathrm{~g}$ del extracto liofilizado de Croton lechleri, se procedió a pesarlo en la balanza analítica; luego se agregó al recipiente el vehículo y se agitó hasta la disolución completa obteniendo una mezcla uniforme. Posteriormente se le agregó el gel de Carbomer 1\% y se mezcló hasta obtener un gel uniforme, se dejó en reposo. Luego se procedió a medir el $\mathrm{pH}$, utilizando el $\mathrm{pH}$ metro calibrado y se obtuvo un promedio de 6,8 para el gel $1 \%$ y 6,9 para el gel 2,5\%. Finalmente se colocaron en envases de vidrio oscuro con sus respectivas etiquetas.

Conformación de los grupos experimentales. Cada grupo estuvo formado por ocho Oryctolagus cuniculus y los subgrupos de tiempo formados por dos animales para experimentar. En cada animal se trabajó en cuatro incisivos: pieza 101 (incisivo superior derecho), 201 (incisivo superior izquierdo), 301 (Incisivo inferior izquierdo), 401 (incisivo inferior derecho).El protocolo para las pulpotomías incluyó: disminución de la carga bacteriana mediante una gasa embebida en clorhexidina al $0,12 \%$ por $30 \mathrm{~s}$. Colocación de anestesia infiltrativa lidocaína al $2 \%$, en el fondo del surco vestibular. Apertura cameral por vestibular del incisivo en el tercio cervical con una fresa redonda diamantada $\mathrm{N}^{\circ} 10$ de alta velocidad, con irrigación de agua para conformar la cavidad. Exéresis total del techo de la cámara pulpar. Amputación de la parte coronal de la pulpa mediante el uso de cureta de dentina afilada $\mathrm{N}^{\circ}$ 45-46. Lavado de la cámara pulpar con una jeringa descartable de $10 \mathrm{ml}$ con cloruro de sodio. Hemostasia con algodón estéril (alcanzada al presionar aproximadamente 1 a 2 min después de emplear un algodón). Comprobación de la total extracción de la pulpa coronal.Colocación de la sustancia sobre el muñón radicular.Grupo control (se colocó óxido de zinc-eugenol). Grupo formocresol (se colocó una bolita embebida en formocresol al 20\% durante $3 \mathrm{~min}$ ). Grupo gel del extracto liofilizado de Croton lechleri 1\% y 2,5\% (se colocó 1 gota de gel del extracto liofilizado de Croton lechlericon un microbrush extrafino blanco $100 \mu$ durante $3 \mathrm{~min}$ ). En todos los grupos se colocó una base de óxido de zinc-eugenol y en la parte superior ionómero de vidrio autocurable de alta viscosidad (Figura 1).
Análisis de variables. Se realizó el seguimiento clínico e histopatológico al cabo de un día, 7, 15, y 30 días, para registrar los signos clínicos como (absceso submucoso, fístula y movilidad dentaria); así como los hallazgos histopatológicos para evaluar la congestión vascular (capilares), presencia de células inflamatorias (polimorfonucleares, linfocitos y macrófagos) y reacción o cambios en el tejido conectivo (fibroblastos, odontoblastos y fibras colágenas).

Luego de un día de tratamiento se eutanatizaron 8 conejos que correspondían: grupo A: 2 conejos, grupo B: 2 conejos, grupo C: 2 conejos y grupo D: 2 conejos. El sacrificio de los animales se llevó a cabo por medio de una sobredosis de $1 \mathrm{~mL}$ de pentobarbital por cada $250 \mathrm{~g}$ de peso del animal, administrado por vía intravenosa? Se empleó el pentobarbital en la presentación de frasco ampolla de $6,5 \mathrm{~g} / 100 \mathrm{~mL}$.

Después de la eutanasia se evaluaron las características clínicas, luego se procedió a desarticular y extraer el maxilar superior y mandíbula del animal, que fue enviada al Departamento de Patología del Hospital María Auxiliadora (Lima. Perú) en frascos de formol al 10\% para el proceso de elaboración de las láminas; las cuales fueron rotuladas con un código para cada muestra,identificadas con el número del animal, tipo de sustancia y número de días en los que fueron trabajados, teniendo dichos cortes histológicos los dientes de los conejos.La lectura de las láminas histológicas se realizó en el Laboratorio de Investigación de la Facultad de Odontología de la Universidad de San Martín de Porres. Se analizó el tercio superior; la evaluación y conteo de las células se realizó por campos. La evaluación fue realizada a través de un Microscopio Binocular Leica DM 750 con cámara incorporada, empleando lentes de 4x, 100x y 400x, para determinar el efecto del gel del extracto liofilizado de Croton lechleri y otros agentes empleados sobre el tejido pulpar en pulpotomías en conejos.

Análisis de datos y permisos éticos. Para el análisis de las variables categóricas se emplearon las frecuencias y se expresaron por medio de un gráfico de barras. Se aplicó la prueba de chi cuadrado y prueba exacta de Fisher. Para el análisis de las variables numéricas se empleó la prueba estadística de ANOVA. Todas las pruebas fueron trabajadas a un nivel de confianza del $95 \%$.Se siguieron todas las normas éticas según la guía de manejo y cuidado de animales de laboratorio: Conejo del Ministerio de Salud, Instituto Nacional de Salud (Lima, Perú), 2018. La presente investigación fue evaluada por el Comité de Ética en Investigación de la Facultad de Medicina de la Universidad Nacional Mayor de San Marcos con código de proyecto: $\mathrm{N}^{\circ} 0350$ y acta $\mathrm{N}^{\circ} 0276$.

\section{Resultados}

En la evaluación a los 1, 7, 15 y 30 días, clínicamente no se evidenció abscesos submucosos, fístulas ni movilidad dentaria en los grupos de tratamiento (control, formocresol, gel del extracto liofilizado de Croton lechleri $1 \%$ y $2,5 \%)$. 


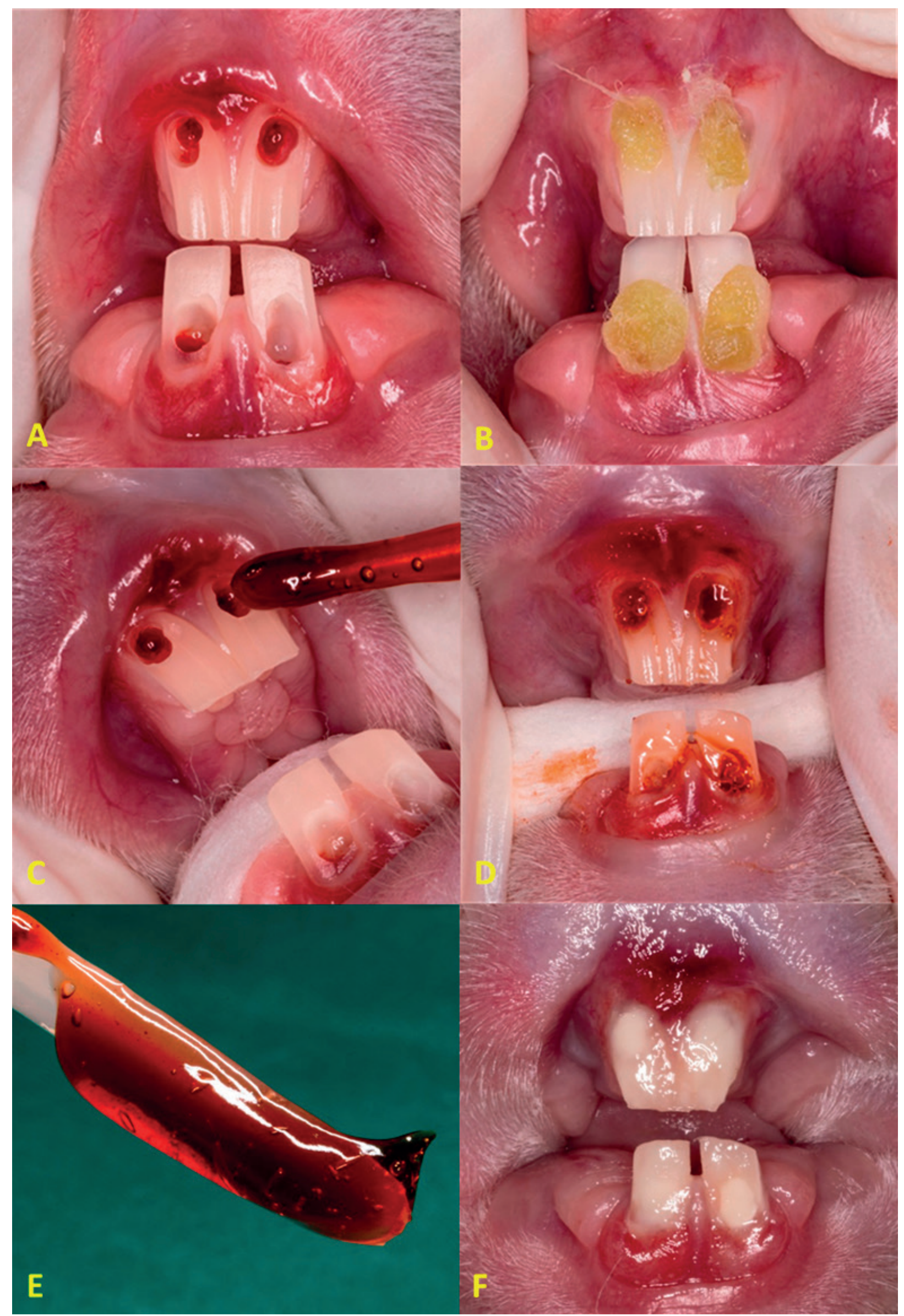

Figura 1. A. Apertura y exéresis de la pulpa cameral en piezas 101, 201, 301, 401. B. Colocación de una bolita embebida en formocresol al $20 \%$ durante $3 \mathrm{~min}$. C. Colocación del gel del extracto liofilizado de Croton lechleri 1\%. D. Colocación del gel del extracto liofilizado de Croton lechleri 2,5\%. E. Consistencia del gel del extracto liofilizado de Croton lechleri.F. Colocación de lonómero de vidrio autocurable de alta viscosidad 
Los resultados histopatológicos evidenciaron que en el primer día, en el grupo control se encontró abundantes capilares, en el grupo formocresol entre moderado y abundante, $\mathrm{n}=4$ en cada categoría y en los grupos gel del extracto liofilizado de Croton lechleri $1 \%$ y $2,5 \%$ moderado, $\mathrm{n}=8$ respectivamente. $(p<0,001)$. A los 7 días en el grupo control se encontró moderada cantidad de capilares, en el grupo formocresol entre leve y abundante, y en los grupos gel del extracto liofilizado de Croton lechleri $1 \%$ y 2,5\% entre leve y moderado. A los 15 días en el grupo control se encontró predominantemente leve, en el grupo formocresol predominantemente abundante, en el grupo gel del extracto liofilizado de Croton lechleri 1\% predominantemente leve y en el grupo gel del extracto liofilizado de Croton lechleri 2,5\% entre leve y moderado. A los 30 días, en el grupo control se encontró predominantemente entre leve y moderado, en el grupo formocresol predominantemente leve y en los grupos gel del extracto liofilizado de Croton lechleri $1 \%$ y $2,5 \%$ predominantemente leve (Figura 2 ).

En la evaluación de los leucocitos polimorfonucleares a 1 día se encontró la mayor cantidad en el grupo gel del extracto liofilizado de Croton lechleri $1 \%$ con una media de 103,06 ( $\mathrm{n}=8)$. A los 7 días se encontró la mayor cantidad en el grupo control 13,77 ( $\mathrm{n}=8)$. Posteriormente

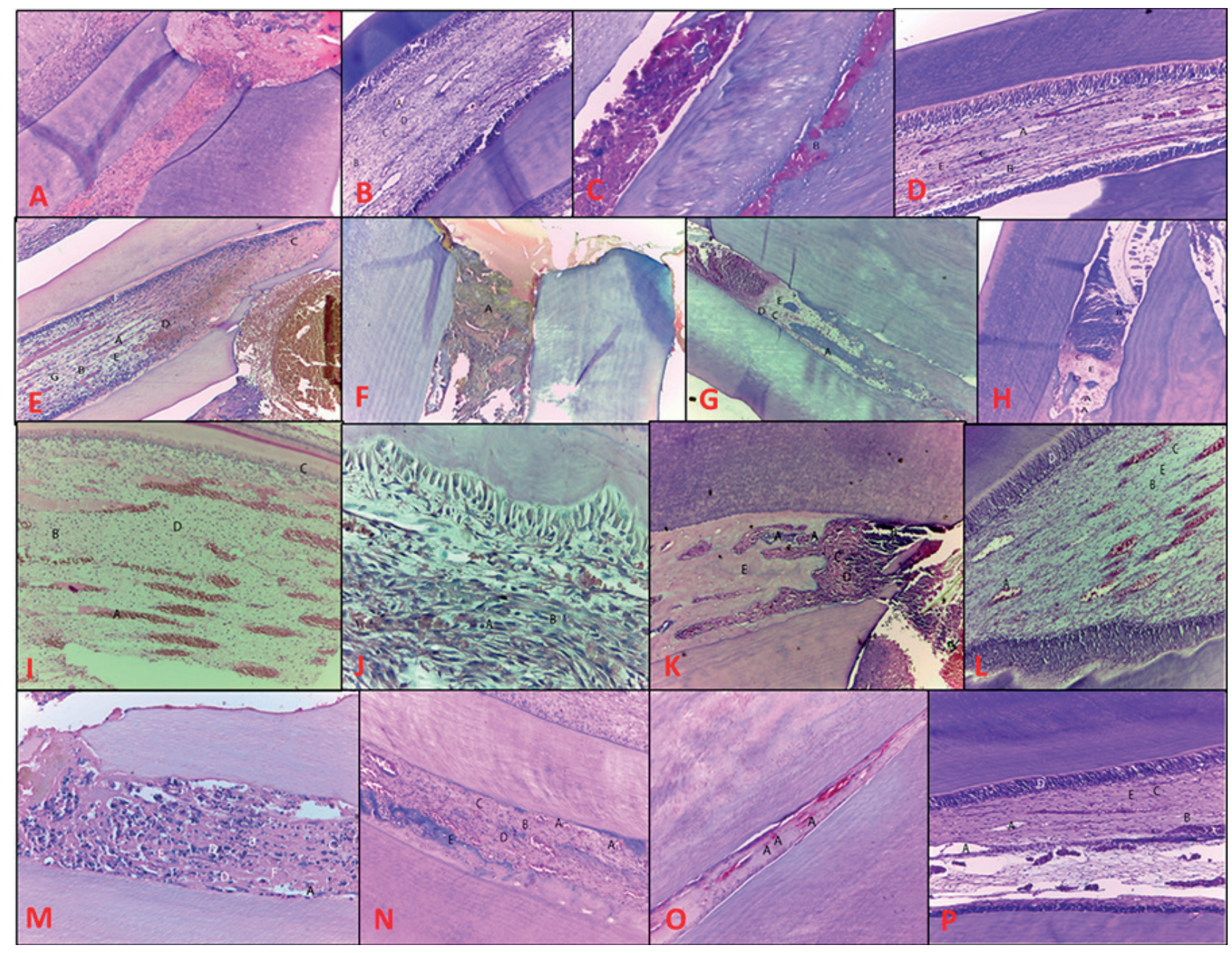

Figura 2. Microfotografías de tejido pulpar (H\&E x100). A. Diente con apertura y exéresis de la pulpa cameral a 1 día con abundante infiltrado leucocitario. B. Apertura y exéresis de la pulpa cameral a los 7 días. (A) Capilares, (B) Linfocitos, (C) Fibroblastos, (D) Fibras colágenas, (E) Odontoblastos. C. Apertura y exéresis de la pulpa cameral a los 15 días. (A) Tejido pulpar destruido, (B) Osteodentina. D. con Apertura y exéresis de la pulpa cameral a los 30 días. (A) Capilares, (B) Linfocitos, (C) Fibroblastos, (D) Odontoblastos, (E) Fibras colágenas. E. Diente pulpotomizado con formocresol a 1 día. F. Diente pulpotomizado con formocresol a los 7 días con (A) pulpa necrótica. G. Formocresol a los 15 días. (A) Capilares, (B) LPMN, (C) Linfocitos, (D) Fibroblastos, (E) Fibras colágenas. H. Formocresol a los 30 días. (A) Capilares, (B) LPMN, C) Linfocitos, (D) Fibroblastos, (E) Fibras colágenas. I. Extracto liofilizado de Croton lechleri 1\% a 1 día. (A) Capilares, (B) Fibroblastos, (C) Odontoblastos, (D) Fibras colágenas; con abundante congestión. J. Extracto liofilizado de Croton lechleri 1\% a los 7 días. Con abundantes: (A) Linfocitos, (B) Fibras colágenas. K. Croton lechleri $1 \%$ a los 15 días. (A) Capilares, (B) LPMN, (C) Linfocitos, (D) Fibras colágenas, (E) Osteodentina. L. Croton lechleri $1 \%$ a los 30 días. (A) Capilares, (B) Linfocitos, (C) Fibroblastos, (D) Odontoblastos, (E) Fibras colágenas. M. Extracto liofilizado de Croton lechleri 2,5\% a 1 día. (A) Capilares, (B) LPMN, (C) Linfocitos, (D) Macrófagos, (E) Fibroblastos. N. Croton lechleri 2,5\% a los 7 días. (A) Capilares, (B) Fibroblastos, (C) Fibras colágenas, (D) Linfocitos, (E) Osteodentina. O. Croton lechleri 2,5\% a los 15 días. (A) Capilares. P. Croton lechleri 2,5\% a los 30 días. (A) Capilares, (B) Linfocitos, (C) Fibroblastos, (D) Odontoblastos (E) Fibras colágenas 
a los 15 días se encontró la mayor cantidad en el grupo control 16,74 $(\mathrm{n}=8)$. Finalmente, a los 30 días se encontró la mayor cantidad en el grupo formocresol 25,66 $(\mathrm{n}=8)$ (Tabla 1).

En la evaluación de los linfocitos a 1 día se encontró la mayor cantidad en el grupo gel del extracto liofilizado de Croton lechleri $1 \%$ con una media de 53,19 ( $\mathrm{n}=8)$. A los 7 días se encontró la mayor cantidad en el grupo gel del extracto liofilizado de Croton lechleri 2,5\% con $5,50(\mathrm{n}=8)$. Posteriormente a los 15 y 30 días se encontró la mayor cantidad en el grupo formocresol con $25,78(\mathrm{n}=8)$ y $8,00(\mathrm{n}=8)$ respectivamente. Respecto a los macrófagos, en el primer día se encontraron en los grupos formocresol y gel del extracto liofilizado de Croton lechleri $1 \%$ entre ausente y leve. A los 7 días en los grupos control y gel del extracto liofilizado de Croton lechleri $2,5 \%$ se encontró entre ausente y moderado, en el grupo formocresol se encontró leve, mientras que en el grupo gel del extracto liofilizado de Croton lechleri 1\% se encontró entre ausente y leve. A los 15 días en el grupo formocresol se encontró entre ausente y moderado, mientras que en el grupo gel del extracto liofilizado de Croton lechleri $1 \%$ se encontró entre ausente y leve. Finalmente, a los 30 días solo se encontró entre ausente y leve en los grupos control y formocresol.

En la evaluación de fibroblastos en todos los tiempos se encontró la mayor cantidad en el grupo gel del extracto liofilizado de Croton lechleri 2,5\%. Al día 1 con una media de 214,25 ( $\mathrm{n}=8)$, a los 7 días 327,03 ( $\mathrm{n}=8)$, a los 15 días 495,16 (n=8) y a los 30 días 585,31(n=8) (Tabla 2).

Respecto a los odontoblastos, a 1 día se encontró la mayor cantidad en los grupos control y gel del extracto liofilizado de Croton lechleri $1 \%$ y $2,5 \%$ con una presencia de $25 \%$. A los 7 días predominó en el grupo gel del extracto liofilizado de Croton lechleri 2,5\% con un $75 \%$. A los 15 días la mayor cantidad se encontró en las muestras del grupo gel del extracto liofilizado de Croton lechleri $2,5 \%$ con un $100 \%$. A los 30 días en los grupos gel del extracto liofilizado de Croton lechleri $1 \%$ y 2,5\% se encontró en todas las muestras 100\%.

Respecto a las fibras colágenas, a 1 día en los grupos control, gel del extracto liofilizado de Croton lechleri $1 \%$ y $2,5 \%$ predominaron fibras colágenas normales, mientras que en el grupo formocresol predominaron alteradas. A los 7 días en los grupos control y formocresol predominaron alterados, mientras en los grupos gel del extracto liofilizado de Croton lechleri 1\% y 2,5\% predominaron normales.A los 15 y 30 días en los grupos control, gel del extracto liofilizado de Croton lechleri $1 \%$ y 2,5\% predominaron normales, mientras que en el grupo formocresol se encontró entre normales y alteradas.

Se evidenció pulpa necrótica en el 100\% de los casos $(\mathrm{n}=8)$ solo en el grupo formocresol a los 7, 15 y 30 días. Otros hallazgos encontrados en mayorcantidad a los 15

Tabla 1. Recuento de leucocitos polimorfonucleares (LPMN) según grupo de tratamiento a los 1, 7, 15 y 30 días

\begin{tabular}{|c|c|c|c|c|c|c|c|}
\hline \multirow{2}{*}{ Tiempo } & \multirow{2}{*}{ Grupos } & \multirow{2}{*}{$\mathbf{n}$} & \multirow{2}{*}{ Media } & \multirow{2}{*}{ DE } & \multicolumn{2}{|c|}{ IC $95 \%$} & \multirow[b]{2}{*}{$\mathrm{p}^{*}$} \\
\hline & & & & & Inferior & Superior & \\
\hline \multirow[t]{5}{*}{1 día } & Control & 8 & 78,58 & 3,740 & 77,21 & 79,95 & \multirow{5}{*}{0,001} \\
\hline & Formocresol & 8 & 24,28 & 16,559 & 18,31 & 30,25 & \\
\hline & Gel de Croton lechleri 1\% & 8 & 103,06 & 99,762 & 67,09 & 139,03 & \\
\hline & Gel de Croton lechleri $2,5 \%$ & 8 & 26,19 & 15,603 & 20,56 & 31,81 & \\
\hline & Total & 32 & 57,87 & 61,196 & 47,12 & 68,61 & \\
\hline \multirow[t]{5}{*}{7 días } & Control & 8 & 13,77 & 13,318 & 8,89 & 18,66 & \multirow{5}{*}{0,013} \\
\hline & Formocresol & 8 & 7,78 & 13,129 & 3,05 & 12,51 & \\
\hline & Gel de Croton lechleri 1\% & 8 & 6,31 & 1,731 & 5,69 & 6,94 & \\
\hline & Gel de Croton lechleri 2,5\% & 8 & 9,06 & 1,435 & 8,54 & 9,58 & \\
\hline & Total & 32 & 9,20 & 9,678 & 7,50 & 10,90 & \\
\hline \multirow[t]{5}{*}{15 días } & Control & 8 & 16,74 & 14,553 & 11,40 & 22,08 & \multirow{5}{*}{0,012} \\
\hline & Formocresol & 8 & 11,19 & 10,126 & 7,54 & 14,84 & \\
\hline & Gel de Croton lechleri 1\% & 8 & 16,22 & 14,823 & 10,87 & 21,56 & \\
\hline & Gel de Croton lechleri 2,5\% & 8 & 16,22 & 14,823 & 10,87 & 21,56 & \\
\hline & Total & 32 & 13,00 & 12,489 & 10,81 & 15,19 & \\
\hline \multirow[t]{5}{*}{30 días } & Control & 8 & 2,74 & 1,966 & 2,02 & 3,46 & \multirow{5}{*}{0,002} \\
\hline & Formocresol & 8 & 25,66 & 24,879 & 16,69 & 34,63 & \\
\hline & Gel de Croton lechleri 1\% & 8 & 1,56 & 840 & 1,26 & 1,87 & \\
\hline & Gel de Croton lechleri 2,5\% & 8 & 1,63 & ,942 & 1,29 & 1,96 & \\
\hline & Total & 32 & 7,94 & 16,137 & 5,10 & 10,77 & \\
\hline
\end{tabular}

*Prueba estadística ANOVA; p<0,05.DE= Desviación estándar. IC. Intervalo de confianza 
Tabla 2. Presencia de fibroblastos según grupo de tratamiento a los 1, 7, 15 y 30 días

\begin{tabular}{|c|c|c|c|c|c|c|c|}
\hline \multirow{2}{*}{ Tiempo } & \multirow{2}{*}{ Grupos } & \multirow{2}{*}{$\mathbf{n}$} & \multirow{2}{*}{ Media } & \multirow{2}{*}{$\mathrm{DE}$} & \multicolumn{2}{|c|}{ IC $95 \%$} & \multirow{2}{*}{$\mathrm{p}^{*}$} \\
\hline & & & & & Inferior & Superior & \\
\hline \multirow[t]{5}{*}{1 día } & Control & 8 & 195,19 & 20,354 & 187,73 & 202,66 & \multirow{5}{*}{0,001} \\
\hline & Formocresol & 8 & 116,69 & 50,263 & 98,57 & 134,81 & \\
\hline & Gel de Croton lechleri $1 \%$ & 8 & 178,75 & 107,185 & 140,11 & 217,39 & \\
\hline & Gel de Croton lechleri 2,5\% & 8 & 214,25 & 94,396 & 180,22 & 248,28 & \\
\hline & Total & 32 & 176,07 & 84,242 & 161,28 & 190,86 & \\
\hline \multirow[t]{5}{*}{7 días } & Control & 8 & 255,84 & 60,506 & 233,64 & 278,03 & \multirow{5}{*}{0,001} \\
\hline & Formocresol & 8 & 254,66 & 102,236 & 217,80 & 291,52 & \\
\hline & Gel de Croton lechleri $1 \%$ & 8 & 216,50 & 145,743 & 163,95 & 269,05 & \\
\hline & Gel de Croton lechleri 2,5\% & 8 & 327,03 & 87,666 & 295,42 & 358,64 & \\
\hline & Total & 32 & 263,57 & 110,365 & 244,19 & 282,95 & \\
\hline \multirow[t]{5}{*}{15 días } & Control & 8 & 390,26 & 62,654 & 367,28 & 413,24 & \multirow{5}{*}{0,002} \\
\hline & Formocresol & 8 & 300,75 & 106,938 & 262,19 & 339,31 & \\
\hline & Gel de Croton lechleri $1 \%$ & 8 & 494,84 & 4,304 & 493,29 & 496,40 & \\
\hline & Gel de Croton lechleri 2,5\% & 8 & 495,16 & 4,112 & 493,67 & 496,64 & \\
\hline & Total & 32 & 420,49 & 102,191 & 402,54 & 438,43 & \\
\hline \multirow[t]{5}{*}{30 días } & Control & 31 & 485,81 & 28,580 & 475,32 & 496,29 & \multirow{5}{*}{0,003} \\
\hline & Formocresol & 8 & 566,25 & 103,977 & 528,76 & 603,74 & \\
\hline & Gel de Croton lechleri $1 \%$ & 8 & 554,19 & 6,916 & 551,69 & 556,68 & \\
\hline & Gel de Croton lechleri 2,5\% & 8 & 585,31 & 70,481 & 559,90 & 610,72 & \\
\hline & Total & 32 & 548,38 & 74,080 & 535,37 & 561,39 & \\
\hline
\end{tabular}

*Prueba estadística ANOVA; < $<0,05 . D E=$ Desviación estándar. IC. Intervalo de confianza

días en el grupo gel del extracto liofilizado de Croton lechleri $2,5 \%$ fue predentina en el 75\% ( $\mathrm{n}=6)$ y osteodentinaen el $100 \%(\mathrm{n}=8)$ de los casos.

\section{Discusión}

En la Odontología contemporánea, el tratamiento pulpar más empleado y controversial en la Odontopediatría es la pulpotomía. Esta terapia emplea diferentes sustancias para preservar el tejido pulpar, siendo la más común el formocresol (FC) que es utilizado desde $1930^{6}$ y que en la actualidad, viene siendo reemplazado por otros biomateriales como el agregado trióxido mineral (MTA) que ha demostrado un efecto preservante y regenerador del tejido pulpar y puede ser una alternativa al formar un puente dentinario delgado ${ }^{7}$; sin embargo, es de alto costo y el tiempo de fraguado es largo, por lo que se han planteado nuevos biomateriales como el sulfato férrico, el silicato tricálcico ${ }^{10} \mathrm{y}$ otros que se basan en plantas tradicionales.

Existen pocas investigaciones sobre pulpotomías en modelos experimentales en animales para observar los efectos clínicos e histopatológicos de los diferentes materiales empleados, lo que se ve reflejadas en la escasa bibliografía relacionada al tema ${ }^{10}$. Es por ello que el presente estudio se enfocó en evaluar los efectos clínicos e histopatológicos del Croton lechleri (sangre de grado) en pulpotomías realizadas en conejos, con evaluaciones de hasta 30 días.
Nuestro estudio encontró que en la evaluación histopatológica, se evidenció menor cantidad de células inflamatorias (leucocitos polimorfonucleares, linfocitos y macrófagos) en el grupo que se administró gel de Croton lechleri $2,5 \%$. Sin embargo, el grupo formocresol presentó mayor recuento de leucocitos polimorfonucleares (LPMN) a los 30 días, recuento de linfocitos a los 15 y 30 días y macrófagos abundantes a los 7 días.Similares resultados observaron Kola et al. ${ }^{11}$, al estudiar 55 niños, reportaron que el formocresol y el hipoclorito de sodio $(\mathrm{NaOCl})$ empleados en 30 pulpotomías por grupo; al evaluarse a los 3 meses encontró que aumentaron la fase de inflamación de leve a moderada (grado 2) con predominio de linfocitos. A los seis meses continuó la inflamación en el grupo $\mathrm{FC}$, mientras que el grupo $\mathrm{NaOCl}$ se estimuló la formación de fibroblastos y colágeno. En ninguna de las muestras del FC encontraron formación del puente de dentina.

En la evaluación clínica, no se encontró la presencia de abscesos submucosos, fístulas o movilidad dentaria en ninguno de los grupos a los 1, 7, 15 y 30 días, similar a la investigación de El Sarek et al. ${ }^{12}$ quienes evaluaron a 37 niños para determinar las características clínicas del formocresol con un total de 112 molares deciduas divididos en dos grupos: Biodentine ${ }^{\mathrm{TM}}(\mathrm{BD}) \mathrm{y}$ formocresol, reportando un porcentaje de éxito clínico al $100 \%$ en ausencia de dolor a la percusión, fistula o absceso y movilidad dentaria a los 3, 6 y 12 meses. Ansari et al. ${ }^{13}$ coinciden en su 
estudio realizado en 40 niños con un total de 160 molares deciduas asignadas al azar. Similar fue el estudio de Junepa et al. ${ }^{14}$ quienes compararon clínicamente los efectos de $\mathrm{BD}, \mathrm{MTA}$ y FC en pulpotomías en molares deciduas de 51 niños entre 5 a 9 años de edad.La tasa de éxito clínico a los 3 meses para las tres técnicas fue $100 \%$, a los 6 meses $\mathrm{BD}$ y $\mathrm{MTA}=100 \%$ y $\mathrm{FC}=80 \%$.

En todos los tiempos evaluados de nuestro estudio, las células de reparación (fibroblastos, odontoblastos y fibras colágenas) fueron significativamente mayores en el grupo gel de Croton lechleri 2,5\%, mostrando un mayor efecto cicatrizante y regenerador en comparación a los otros tratamientos. Estos resultados son similares a los reportados por Cavalie et al. ${ }^{15}$, quienes evidenciaron la efectividad cicatrizante del Croton lechleri puro aplicado tópicamente en el alveolo post exodoncia de 50 pacientes entre 20 y 60 años; presentando cicatrización al $100 \%$ de la herida a los 18,3 días en el grupo experimental y 27,1 días en el grupo control. Asimismo, en el grupo experimental el tiempo promedio de desaparición de la inflamación fue de 2,2 días y del grupo control fue 3,8 días.

El estudio de Oliadarani et al. ${ }^{16}$, evaluaron la reacción de la pulpa dental al aplicar pasta de óxido de zinc como agente de recubrimiento después de la pulpotomía con formocresol en ocho caninos deciduos, se observó inflamación leve después de 1 mes de seguimiento. Asimismo, Maldonado ${ }^{17}$, comparó la respuesta pulpar en 52 dientes de conejo aplicando formocresol e hipoclorito de sodio 5\% a los 7, 14 y 30 días, encontrando que el grupo al que se aplicó formocresol evidenció la mayor cantidad de células inflamatorias severas (LPMN), así como áreas de necrosis leve en el sitio de reacción en todos los tiempos evaluados. Similares resultados encontramos en la presente investigación con presencia de pulpa necrótica en todas las muestras en el grupo que se aplicó formocresol a los 7, 15 y 30 días.

Cevallos et al.$^{18}$, determinaron la actividad cicatrizante y toxicidad de Croton lechleri luego de aplicar dos gotas del látex diluido 1:10 con agua destilada; sobre incisiones de $10 \mathrm{~mm}$ en el lomo de 15 ratas Wistar comparada con la aplicación de una crema farmacéuticay un grupo sin tratamiento a los 7 días. Con la aplicación del látex se observó tejido de reparación (fibroblastos), menor tamaño de la incisión $(6,8 \mathrm{~mm})$ y formación de costras en un tiempo menor a 2,8 días; mientras que las otras ratas manifestaron superficies ulcerosas rodeadas con exudado purulento y se formó costras a los 6 y 7 días respectivamente. En la evaluación de la toxicidad aguda se aplicó una dosis de látex $2000 \mathrm{mg} / \mathrm{kg}$ de peso a cada animal por 14 días, no manifestando toxicidad a esta dosis. Estos resultados coinciden con la presente investigación, debido a que en todos los tiempos evaluados las células de reparación (fibroblastos y odontoblastos) fueron mayores y las fibras colágenas fueron normales en el grupo que se aplicó gel del extracto liofilizado de Croton lechleri 2,5\%. Así mismo, al realizar la prueba de dosis letal, no se observó ningún signo de toxicidad en el gel del extracto liofilizado de Croton lechleri 1\% y 2,5\%.
En la actualidad no existen estudios sobre geles de uso tópico a base de Croton lechleri en procedimientos de odontología y nos guiamos del estudio de Gallardo ${ }^{19}$, quien empleó 15 ratones en la elaboración de geles del látex de Croton lechleri en diferentes concentraciones $(0,5 \%, 1 \%$ y $2 \%)$, encontró un efecto cicatrizante con el gel a una concentración de $2 \%$ presentando efecto dosis-dependiente. Sus resultados concuerdan con la presente investigación al elaborar geles $1 \%$ y $2,5 \%$ en base a las características de solubilidad del extracto liofilizado de Croton lechleri; encontramos que el mayor efecto cicatrizante lo presentó el extracto liofilizado de Croton lechleri al 2,5\%, esto se debería a que el principal componente de la sangre de grado es el alcaloide taspina y su mecanismo de acción está relacionado con la estimulación de los fibroblastos y el inicio del proceso de cicatrización en las primeras 60 horas tal como lo refiere Planas ${ }^{20}$ y Milla ${ }^{21}$.

Otro hallazgo relevante del presente estudio fue encontrar a los 7 días predentina en el 50\% de los casos en los grupos que se administró gel del extracto liofilizado de Croton lechleri $1 \%$ y $2,5 \%$. A los 15 días se evidenció $62,5 \%$ de los casos en el grupo gel del extracto liofilizado de Croton lechleri 1\%, y en el 75\% de los casos en el grupo gel del extracto liofilizado de Croton lechleri $2,5 \%$. Estos hallazgos fueron similares a los reportados por Ratnakumari et al. ${ }^{22}$ en el comportamiento del tejido pulpar de 20 caninos deciduos de niños entre 10 y 12 años, frente al formocresol y Chitra-Cemento de fosfato de calcio (Chitra-CPC) en dientes extraídos después de 70 días. La puntuación fue de 1,6 para Chitra-CPC y 2,6 para formocresol, en la formación del puente de dentina.

La presente investigación propone al gel del extracto liofilizado de Croton lechleri 2,5\% como un compuesto alternativo a los materiales existentes en el mercado, por presentar resultados clínicos e histopatológicos favorables en pulpotomía en conejos, evidenciando efectos cicatrizantes, antiinflamatorios y regeneradores, sin evidencia de daño pulpar. Además, sería un material a un bajo costo, siendo una alternativa por ser un producto natural alternativo a la odontología convencional, a la vez que puede ser incorporado en investigaciones en la práctica clínica odontopediátrica. Se recomienda que en futuros trabajos de investigación se considere el uso del gel del extracto liofilizado de Croton lechleri 2,5\% en ensayos clínicos aleatorizados para evaluar su eficacia. Además, compararlos con otras sustancias como el sulfato férrico, MTA y silicato tricálcico. Asimismo, realizar estudios de costo-efectividad del uso del gel del extracto liofilizado de Croton lechleri 2,5\% en el tratamiento de pulpotomías.

Concluimos que el gel del extracto liofilizado de Croton lechleri evidenció mayor efecto antiinflamatorio, cicatrizante y regenerador sobre el tejido pulpar radicular en comparación a los otros tratamientos. Al comparar las tres sustancias empleadas en la evaluación clínica, en todos los tiempos evaluados no se evidenciaron alteraciones. El grupo formocresol presentó abundante 
congestión vascular en todos los tiempos evaluados, así como pulpa necrótica a los 7, 15 y 30 días. El gel del extracto liofilizado de Croton lechleri 2,5\% presentó mayor cantidad de células de reparación en todos los tiempos evaluados; además se encontró predentina y osteodentina en mayor cantidad a los 15 días.

\section{Referencias bibliográficas}

1. Ministerio de Salud. Oficina General de Tecnología de la Información. Lima: Ministerio de Salud; 2017.

2. Bezerra LA. Tratado de Odontopediatría. 2a ed. Bogotá: Amolca; 2018.

3. Bordoni N, Escobar A, Castillo R. Odontología Pediátrica. La salud bucal del niño y el adolescente en el mundo actual. $1^{\text {a }}$ ed. Buenos Aires: Médica Panamericana; 2010.

4. Nadin G, Goel BR, Yeung CA, Glenny AM. Pulp treatment for extensive decay in primary teeth. Cochrane Database Syst Rev. 2003;(1):CD003220.

5. Rojas $S$. Terapias pulpares en dientes temporales ¿ueva era de terapias pulpares? Rev Soc Chil Odontopediatría. 2011;26(2):4-12.

6. Vaisberg AJ, Milla M, Planas MC, Córdova JL, Rosas E, Ferreyra R, et al. Taspine is the cicatrizant principle in Sangre de Grado extracted from Croton lecheri.Planta Med. 1989;55:140-3.

7. Marcelo AJ, Meza EN. Propiedades Biológicas de metabolitos secundarios de "Sangre de Grado". En: Marcelo AJ, Calderón C, Medina D, Valencia M, Pariona M, Meza EN. Desarrollando nuestra diversidad biocultural: "Sangre de grado" y el reto de su producción sustentable en el Perú. Lima: Universidad Nacional Mayor de San Marcos; 1999. p. 165-96.

8. Lazo J. Extracto de Croton lechleri y de Pelargonium robertianum $\mathrm{L}$. en el tratamiento de la gingivitis asociada al embarazo. Kiru. 2007;4(2):52-9.

9. Committee for the Update of the Guide for the Care and Use of Laboratory Animals. Guide for the care and use of laboratory animals. Washington, DC; 2011. https:// grants.nih.gov/grants/olaw/guide-for-the-care-and-useof-laboratory-animals.pdf.

10. American Academy of Pediatric Dentistry. Guideline on pulp therapy for primary and immature permanent teeth. Reference Manual 2009-10. Pediatr Dent. 2009;31:179-84

11. Kola SR, Reddy NV, Sneha T, Reddy MA, Niharika P, Kumar PJ. A histopathological comparison of pulpal response to formocresol and sodium hypochlorite used as pulpotomy medicaments: In primary teeth A clinical clinical trial. J Indian Soc Pedod Prev Dent. 2020;37(12):198-204.
12. El Sadek O, Mohamed N, Mohamed S, Monhsen A. Biodentine $^{\mathrm{TM}}$ versus formocresol pulpotomy technique in primary molars: a $12-$ month randomized controlled clinical trial. BMC Oral Health. 2019;19(3):2-8.

13. Ansari G. Evaluation of four pulpotomy techniques in primary molars: A Randomized Controlled Trial. Iran End J. 2018;13(1):7-12.

14. Juneja P, Kulkarni S. Clinical and radiographic comparison of Biodentine ${ }^{\mathrm{TM}}$ mineral trioxide aggregate and formocresol as pulpotomy agents in primary molars. Eur Arch Paediatr Dent. 2017;18:271-8.

15. Cavalie K, Ortega M, Basilio Y. Efectividad de la sangre de grado Croton lechleri en la cicatrización de heridas post exodoncia en adultos. Rev Inv Val. 2018;13(1):7-14.

16. Oliadarani FK, Haghgoo R, Mashhadiabbas F, Kahvand M. Histopathological evaluation of dental pulp of primary teeth pulpotomized with formocresol with/without a capping agent: A randomized clinical trial. J Int Soc Prevent Communit Dent. 2018;8(10):420-3.

17. Maldonado LA. Respuesta histológica de la pulpa dental con formocresol 1:5 e hipoclorito de sodio al 5\% en dientes pulpotomizados de Oryctolagus cuniculus [tesis de licenciatura]. [Lima]: Facultad de Odontología, Universidad Nacional Mayor de San Marcos; 2016. 85 p.

18. Cevallos D, Jaramillo C, Cuesta O, Cuesta O, Zaldua J, García G, et al. Composición química, actividad cicatrizante y toxicidad del látex Croton lechleri. Rev Cient. 2016;26(2):95-103.

19. Gallardo G. Efecto cicatrizante del gel elaborado del látex de Croton lechleri "Sangre de Drago".Rev Cient Cienc Med. 2015;18(1):10-6.

20. Planas MC. Caracterización de la actividad biológica del alcaloide taspina del látex de Croton lechleri M. Arg. (Sangre de grado) [tesis de licenciatura]. [Lima]: Facultad de Ciencias Biológicas, Universidad Peruana Cayetano Heredia; 1984.112 p.

21. Milla ME. Estudio sobre el mecanismo de acción del principio activo de la "Sangre de Grado". [Tesis de licenciatura]. [Lima]: Facultad de Ciencias Biológicas, Universidad Peruana Cayetano Heredia; 1985. 100 p.

22. Ratnakumari N, Thomas B. A Histopathological comparison of pulpal response to Chitra-CPC and Formocresol used as pulpotomy agents in primary teeth: A Clinical Trial. Int J Clin Pediatr Dent. 2012;5(1):6-13. 